\title{
Existence and multiplicity of positive solutions for a nonlocal problem
}

\author{
Yu Duan ${ }^{a}$, Xin Sun ${ }^{a}$, Hong-Ying Li $i^{\text {b,* }}$ \\ ${ }^{a}$ College of Science, Guizhou University of Engineering Science, Bijie Guizhou 551700, People's Republic of China. \\ ${ }^{b}$ School of Mathematics and Information, China West Normal University, Nanchon, Sichuan 637002, People's Republic of China.
}

Communicated by K. Q. Lan

\begin{abstract}
In this work, we are interested in considering the following nonlocal problem

$$
\left\{\begin{array}{l}
-\left(a-b \int_{\Omega}|\nabla u|^{2} d x\right) \Delta u=f(x)|u|^{p-2} u, \quad \text { in } \Omega \\
u=0, \quad \text { on } \partial \Omega
\end{array}\right.
$$

where $\Omega \subset \mathbb{R}^{N}(N \geqslant 3)$ is a bounded domain with smooth boundary $\partial \Omega, a, b>0,1 \leqslant p<2^{*}, f \in L^{\frac{2^{*}}{2^{*}}-p}(\Omega)$ is nonzero and nonnegative. By using the variational method, some existence and multiplicity results are obtained. (C)2017 All rights reserved.
\end{abstract}

Keywords: Nonlocal problem, positive solutions, variational method.

2010 MSC: 35B09, 35J60, 35J67.

\section{Introduction and main result}

In this paper, we consider the following nonlocal problem

$$
\left\{\begin{array}{l}
-\left(a-b \int_{\Omega}|\nabla u|^{2} d x\right) \Delta u=f(x)|u|^{p-2} u, \quad \text { in } \Omega, \\
u=0, \quad \text { on } \partial \Omega
\end{array}\right.
$$

where $\Omega \subset \mathbb{R}^{N}(N \geqslant 3)$ is a bounded domain with smooth boundary $\partial \Omega, a, b>0,1 \leqslant p<2^{*}$, the weight function $f \in \mathrm{L}^{\frac{2^{*}}{2^{*}}}(\Omega)$ is nonzero and nonnegative. $2^{*}=\frac{2 \mathrm{~N}}{\mathrm{~N}-2}$ is the critical Sobolev exponent for the embedding of $\mathrm{H}_{0}^{1}(\Omega)$ into $\mathrm{L}^{\mathrm{q}}(\Omega)$ for every $\mathrm{q} \in\left[1,2^{*}\right]$, where $\mathrm{H}_{0}^{1}(\Omega)$ is a Sobolev space equipped with the norm $\|\mathfrak{u}\|=\left(\int_{\Omega}|\nabla \mathfrak{u}|^{2} \mathrm{dx}\right)^{\frac{1}{2}}$, and $|\mathfrak{u}|_{\mathfrak{q}}=\left(\int_{\Omega}|\mathfrak{u}|^{\mathfrak{q}}\right)^{\frac{1}{q}}$ denotes the norm of $\mathrm{L}^{\mathrm{q}}(\Omega)$.

When $2<p<2^{*}$ and $f(x) \equiv 1$, problem (1.1) was considered by [5] for the first time. By using the mountain pass lemma, they obtained the existence of nontrivial solutions for problem (1.1). One of their

\footnotetext{
*Corresponding author

Email address: lihongyingnch@163.com (Hong-Ying Li)
}

doi:10.22436/jnsa.010.11.40 
important works is the $(\mathrm{PS})_{\mathrm{c}}$ condition with $\mathrm{c}<\frac{\mathrm{a}^{2}}{\mathrm{bb}}$. Recently, [3] studied problem (1.1) with $\mathrm{N}=3$ and $1<p<2$. When $f \in L^{\infty}(\Omega)$ changes sign, they got two positive solutions by the variational method and Harnack inequality. Compared with [5], they used a different method to prove the $(P S)_{c}$ condition with $\mathrm{c}<\frac{\mathrm{a}^{2}}{\mathrm{ub}}$. While $0<\mathrm{p}<1$ and $\mathrm{N}=3$, problem (1.1) was researched by [2].

Inspired by the works in [2,3] and [5], we study the existence and multiplicity of positive solutions for problem (1.1) with $N \geqslant 3$ and $1 \leqslant p<2^{*}$. Via the variational method and strong maximum principle, when $1 \leqslant p<2$, we obtain two positive solutions of problem (1.1); while $2 \leqslant p \leqslant 2^{*}$, we get the existence of positive solutions of problem (1.1). Our results generalize and complete the results of [3] and [5].

The energy functional corresponding to problem (1.1) is given by

$$
\mathrm{I}(\mathrm{u})=\frac{\mathrm{a}}{2}\|\mathrm{u}\|^{2}-\frac{\mathrm{b}}{4}\|\mathrm{u}\|^{4}-\frac{1}{\mathrm{p}} \int_{\Omega} \mathrm{f}(\mathrm{x})|\mathrm{u}|^{\mathrm{p}} \mathrm{d} x, \quad \forall \mathrm{u} \in \mathrm{H}_{0}^{1}(\Omega) .
$$

In general, a function $u$ is called a weak solution of problem (1.1) if $u \in H_{0}^{1}(\Omega)$ and for all $\varphi \in H_{0}^{1}(\Omega)$ it holds

$$
\left(a-b\|u\|^{2}\right) \int_{\Omega}(\nabla u, \nabla \varphi) d x-\int_{\Omega} f(x)|u|^{p-2} u \varphi d x=0 .
$$

Let $S$ be the best Sobolev constant, namely

$$
S:=\inf _{u \in D^{1,2}\left(\mathbb{R}^{N}\right) \backslash\{0\}} \frac{\int_{\mathbb{R}^{N}}|\nabla u|^{2} \mathrm{~d} x}{\left(\int_{\mathbb{R}^{N}}|u|^{2^{*}} \mathrm{~d} x\right)^{\frac{2^{2 *}}{2}}}:=\inf _{u \in H_{0}^{1}(\Omega) \backslash\{0\}} \frac{\int_{\Omega}|\nabla u|^{2} \mathrm{~d} x}{\left(\int_{\Omega}|u|^{2^{*}} \mathrm{~d} x\right)^{\frac{2}{2^{*}}}} .
$$

Now our main result can be described as follows:

Theorem 1.1. Assume that $\mathrm{a}, \mathrm{b}>0,1 \leqslant \mathrm{p}<2^{*}$ and $\mathrm{f} \in \mathrm{L}^{2^{2^{*}-\mathrm{p}}}(\Omega)$ is nonzero and nonnegative, then

(1) when $1 \leqslant p<2$, there exists $\mathrm{T}>0$ such that for any $|\mathrm{f}|_{\frac{2^{*}}{2^{*}-\mathrm{p}}}<\mathrm{T}$, (1.1) has at least two positive solutions $\mathfrak{u}_{*}, \mathfrak{u}_{* *}$ with $\mathrm{I}\left(\mathfrak{u}_{*}\right)<0$ and $\mathrm{I}\left(\mathfrak{u}_{* *}\right)>0$;

(2) when $\mathrm{p}=2,|\mathrm{f}|_{2^{*}}<\mathrm{aS}$ or $2<\mathrm{p}<2^{*}$, (1.1) has at least one positive mountain-pass solution $\mathrm{u}_{* *}$ with $\mathrm{I}\left(\mathrm{u}_{* *}\right)>0$.

Remark 1.2. Compared with [3] and [5], we consider (1.1) with $p=1,2$ and obtain the existence of positive solutions by the strong maximum principle. Particular, compared with [5], we study problem (1.1) with $1 \leqslant p \leqslant 2$ and obtain the existence and multiplicity of positive solutions. Compared with [3], we generalize the dimension $N=3$ to $N \geqslant 3$.

\section{Proof of Theorem 1.1}

In this part, we will give the proof of Theorem 1.1. Before proving Theorem 1.1, we give the following lemma.

Lemma 2.1. Assume $a, b>0,1 \leqslant p<2^{*}$ and $f \in L^{\frac{2^{*}}{2^{*}-p}}(\Omega)$ is nonzero and nonnegative, then I satisfies the $(\mathrm{PS})_{\mathrm{c}}$ condition with $\mathrm{c}<\frac{\mathrm{a}^{2}}{4 \mathrm{~b}}$.

Proof. Suppose $\left\{u_{n}\right\} \subset H_{0}^{1}(\Omega)$ is a (PS $)_{c}$ sequence for I, that is,

$$
\mathrm{I}\left(\mathrm{u}_{\mathrm{n}}\right) \rightarrow \mathrm{c}, \quad \mathrm{I}^{\prime}\left(\mathrm{u}_{\mathrm{n}}\right) \rightarrow 0, \quad \text { as } \mathrm{n} \rightarrow \infty .
$$

By the Hölder inequality and (1.3), one has

$$
\int_{\Omega} f(x)|u|^{p} d x \leqslant|f|_{\frac{2^{*}}{2^{*}-p}}|u|_{2^{*}}^{p} \leqslant|f|_{\frac{2^{*}}{2^{*}-p}} S^{-\frac{p}{2}}\|u\|^{p} .
$$


When $1 \leqslant p<2$, it follows from (2.1) and (2.2) that

$$
\begin{aligned}
1+c+o\left(\left\|u_{n}\right\|\right) & \geqslant I\left(u_{n}\right)-\frac{1}{4}\left\langle I^{\prime}\left(u_{n}\right), u_{n}\right\rangle \\
& =\frac{a}{4}\left\|u_{n}\right\|^{2}-\frac{4-p}{4 p} \int_{\Omega} f(x)\left|u_{n}\right|^{p} d x \\
& \geqslant \frac{a}{4}\left\|u_{n}\right\|^{2}-\frac{(4-p)|f| \frac{2^{*}}{2^{*}-p}}{4 p S^{\frac{p}{2}}}\left\|u_{n}\right\|^{p},
\end{aligned}
$$

which implies that $\left\{u_{n}\right\}$ is bounded in $H_{0}^{1}(\Omega)$. When $2 \leqslant p<2^{*}$, it follows from (2.1) that

$$
\begin{aligned}
1+c+o\left(\left\|u_{n}\right\|\right) & \geqslant I\left(u_{n}\right)-\frac{1}{2}\left\langle I^{\prime}\left(u_{n}\right), u_{n}\right\rangle \\
& =\frac{b}{4}\left\|u_{n}\right\|^{4}+\frac{p-2}{2 p} \int_{\Omega} f(x)\left|u_{n}\right|^{p} d x \\
& \geqslant \frac{b}{4}\left\|u_{n}\right\|^{4},
\end{aligned}
$$

which implies that $\left\{u_{n}\right\}$ is bounded in $H_{0}^{1}(\Omega)$. Going if necessary to a subsequence, still denoted by $\left\{u_{n}\right\}$, there exists $u \in H_{0}^{1}(\Omega)$ such that

$$
\begin{cases}u_{n} \rightarrow u, & \text { weakly in } H_{0}^{1}(\Omega), \\ u_{n} \rightarrow u, & \text { strongly in } L^{s}(\Omega), 1 \leqslant s<2^{*}, \\ u_{n}(x) \rightarrow u(x), & \text { a.e. in } \Omega,\end{cases}
$$

as $n \rightarrow \infty$. Moreover, by the Vitali Theorem, one obtains

$$
\lim _{n \rightarrow \infty} \int_{\Omega} f(x)\left|u_{n}\right|^{p} d x=\int_{\Omega} f(x)|u|^{p} d x
$$

Set $w_{n}=u_{n}-u$, then $\left\|w_{n}\right\| \rightarrow 0$. Otherwise, there exists a subsequence, still denoted by $\left\{w_{n}\right\}$, such that

$$
\lim _{n \rightarrow \infty}\left\|w_{n}\right\|=l>0
$$

From (2.1), for every $\phi \in \mathrm{H}_{0}^{1}(\Omega)$, it holds

$$
\left(a-b\left\|u_{n}\right\|^{2}\right) \int_{\Omega}\left(\nabla u_{n}, \nabla \phi\right) d x-\int_{\Omega} f(x)\left|u_{n}\right|^{p-2} u_{n} \phi d x=o(1) .
$$

Letting $n \rightarrow \infty$, by using (2.3), we have

$$
\left(a-b l^{2}-b\|u\|^{2}\right) \int_{\Omega}(\nabla u, \nabla \phi) d x-\int_{\Omega} f(x)|u|^{p-2} u \phi d x=0 .
$$

Taking $\phi=u$ in (2.4), one has

$$
\left(a-b l^{2}-b\|u\|^{2}\right)\|u\|^{2}-\int_{\Omega} f(x)|u|^{p} d x=0 .
$$

Note that $\left\langle\mathrm{I}^{\prime}\left(u_{n}\right), u_{n}\right\rangle \rightarrow 0$ as $n \rightarrow \infty$, it holds

$$
a\left\|w_{n}\right\|^{2}+a\|u\|^{2}-b\left\|w_{n}\right\|^{4}-2 b\left\|w_{n}\right\|^{2}\|u\|^{2}-b\|u\|^{4}-\int_{\Omega} f(x)|u|^{p} d x=o(1) .
$$


It follows from (2.5) and (2.6) that

$$
\mathrm{a}\left\|w_{\mathrm{n}}\right\|^{2}-\mathrm{b}\left\|w_{\mathrm{n}}\right\|^{4}-\mathrm{b}\left\|w_{\mathrm{n}}\right\|^{2}\|\mathrm{u}\|^{2}=\mathrm{o}(1) .
$$

Consequently, one has $l^{2}\left(a-b\|u\|^{2}-b l^{2}\right)=0$, that is,

$$
l^{2}=\frac{a}{b}-\|u\|^{2} .
$$

On the one hand, from (2.5) and (2.8), we have

$$
\begin{aligned}
I(u) & =\frac{a}{2}\|u\|^{2}-\frac{b}{4}\|u\|^{4}-\frac{1}{p} \int_{\Omega} f(x)|u|^{p} d x \\
& =\frac{a}{2}\|u\|^{2}-\frac{b}{4}\|u\|^{4}-\frac{1}{p}\left(a\|u\|^{2}-b l^{2}\|u\|^{2}-b\|u\|^{4}\right) \\
& =\frac{a(p-2)}{2 p}\|u\|^{2}+\frac{b(4-p)}{4 p}\|u\|^{4}+\frac{b}{p}\|u\|^{2} \frac{a-b\|u\|^{2}}{b} \\
& =\frac{a}{2}\|u\|^{2}-\frac{b}{4}\|u\|^{4} .
\end{aligned}
$$

On the other hand, by (2.1), (2.7) and (2.8), it follows from $c<\frac{a^{2}}{4 b}$ that

$$
\begin{aligned}
I(u) & =\lim _{n \rightarrow \infty}\left[I\left(u_{n}\right)-\frac{a}{2}\left\|w_{n}\right\|^{2}+\frac{b}{4}\left\|w_{n}\right\|^{4}+\frac{b}{2}\left\|w_{n}\right\|^{2}\|u\|^{2}\right] \\
& =\lim _{n \rightarrow \infty}\left[I\left(u_{n}\right)-\frac{a}{2}\left\|w_{n}\right\|^{2}+\frac{b}{4}\left\|w_{n}\right\|^{4}+\frac{1}{2}\left(a\left\|w_{n}\right\|^{2}-b\left\|w_{n}\right\|^{4}\right)\right] \\
& =c-\frac{b}{4} l^{4} \\
& =c-\frac{a^{2}}{4 b}+\frac{a}{2}\|u\|^{2}-\frac{b}{4}\|u\|^{4} \\
& <\frac{a}{2}\|u\|^{2}-\frac{b}{4}\|u\|^{4},
\end{aligned}
$$

which contradicts (2.9). Hence, $l \equiv 0$, that is, $u_{n} \rightarrow u$ in $H_{0}^{1}(\Omega)$ as $n \rightarrow \infty$. Therefore, I satisfies the (PS) condition for $c<\frac{a^{2}}{4 b}$. This completes the proof of Lemma 2.1 .

Now, we give the following two important propositions.

Proposition 2.2. Assume $1 \leqslant p<2$ and $f \in \mathrm{L}^{\frac{2^{*}}{2^{*}-p}}(\Omega)$ is nonzero and nonnegative. There exists $T>0$ such that for any $|f|_{\frac{2^{*}}{2^{*}-p}}<T,(1.1)$ has at least one positive local minimal solution $\mathfrak{u}_{*}$ with $\mathrm{I}\left(\mathfrak{u}_{*}\right)<0$.

Proof. We claim that there exist $T, R, \rho>0$ such that for every $|f|_{\frac{2^{*}}{2^{*}-p}}<T$, I satisfies

$$
\left.\mathrm{I}(\mathrm{u})\right|_{\mathfrak{u} \in \mathrm{S}_{\mathrm{R}}} \geqslant \rho, \inf _{\mathfrak{u} \in \mathrm{B}_{\mathrm{R}}} \mathrm{I}_{\lambda}(\mathrm{u})<0,
$$

where $B_{R}=\left\{u \in H_{0}^{1}(\Omega):\|u\| \leqslant R\right\}$ is a closed ball and $S_{R}=\left\{u \in H_{0}^{1}(\Omega):\|u\|=R\right\}$. It follows from (2.2) that

$$
\begin{aligned}
& I(u)=\frac{a}{2}\|u\|^{2}-\frac{b}{4}\|u\|^{4}-\frac{1}{p} \int_{\Omega} f(x)|u|^{p} d x \\
& \geqslant \frac{a}{2}\|u\|^{2}-\frac{b}{4}\|u\|^{4}-\frac{|f|_{2^{*}-p}}{p S^{\frac{p}{2}}}\|u\|^{p} \\
& =\|u\|^{p}\left(\frac{a}{2}\|u\|^{2-p}-\frac{b}{4}\|u\|^{4-p}-\frac{|f|_{\frac{2^{*}}{2^{*}-p}}}{p S^{\frac{p}{2}}}\right) .
\end{aligned}
$$


For any $t \geqslant 0, g(t)$ is defined by

$$
g(t)=\frac{a}{2} t^{2-p}-\frac{b}{4} t^{4-p}-\frac{|f| \frac{2^{*}}{2^{*}-p}}{p S^{\frac{p}{2}}}
$$

then

$$
g^{\prime}(t)=t^{1-p}\left[\frac{a(2-p)}{2}-\frac{b(4-p)}{4} t^{2}\right]
$$

Consequently, let $g^{\prime}(t)=0$, we can easily get $t_{\max }=\left[\frac{2 a(2-p)}{b(4-p)}\right]^{\frac{1}{2}}$ such that

$$
\max _{t \geqslant 0} g(t)=g\left(t_{\max }\right)=\frac{a}{4-p}\left[\frac{2 a(2-p)}{b(4-p)}\right]^{\frac{2-p}{2}}-\frac{|f|_{\frac{2^{*}}{2^{*}-p}}}{p S^{\frac{p}{2}}} .
$$

Choosing

$$
\mathrm{T}=\frac{a p S^{\frac{p}{2}}}{4-p}\left[\frac{2 a(2-p)}{b(4-p)}\right]^{\frac{2-p}{2}}, \quad R=\left[\frac{2 a(2-p)}{b(4-p)}\right]^{\frac{1}{2}},
$$

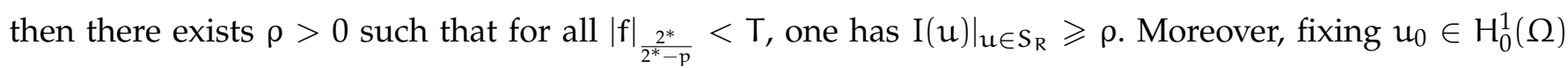
and $u_{0} \neq 0$, one gets

$$
\lim _{t \rightarrow 0^{+}} \frac{I\left(t u_{0}\right)}{t^{p}}=-\frac{1}{p} \int_{\Omega} f(x)\left|u_{0}\right|^{p} d x<0 .
$$

Thus, one has $\inf _{\mathfrak{u} \in \mathrm{B}_{\mathrm{R}}} \mathrm{I}(\mathfrak{u})<0$. Therefor, our claim is true. Without loss of generality, we denote

$$
m=\inf _{u \in B_{R}} I(u)
$$

For this minimization problem, there exists a minimization sequence $\left\{u_{n}\right\}$ such that $\lim _{n \rightarrow \infty} I\left(u_{n}\right)=m$. Moreover, by [4, Proposition 9], we can take a subsequence from $\left\{u_{n}\right\}$, still denotes by $\left\{u_{n}\right\}$, such that $\left\{u_{n}\right\}$ is a (PS $)_{m}$ sequence of $I$ in $H_{0}^{1}(\Omega)$. Thus, by Lemma 2.1, there exists $u_{*} \in H_{0}^{1}(\Omega)$ such that $u_{n} \rightarrow u_{*}$ in $\mathrm{H}_{0}^{1}(\Omega)$ as $\mathrm{n} \rightarrow \infty$ and $\mathrm{I}\left(\mathrm{u}_{*}\right)=\mathrm{m}<0$. Consequently, $\mathrm{u}_{*}$ is a nonzero solution of problem (1.1). Since $\mathrm{I}(\mathfrak{u})=\mathrm{I}(|\mathfrak{u}|)$, we can assume that $\mathfrak{u}_{*} \geqslant 0$ in $\Omega$. From (1.2), choosing $\varphi=\mathfrak{u}_{*}$, we have

$$
\left(a-b\left\|u_{*}\right\|^{2}\right) \int_{\Omega}\left|\nabla u_{*}\right|^{2} d x-\int_{\Omega} f(x)\left|u_{*}\right|^{p} d x=0
$$

Consequently, one has

$$
\left(a-b\left\|u_{*}\right\|^{2}\right) \int_{\Omega}\left|\nabla u_{*}\right|^{2} d x=\int_{\Omega} f(x)\left|u_{*}\right|^{p} d x \geqslant 0
$$

which implies that

$$
a-b\left\|u_{*}\right\|^{2} \geqslant 0
$$

Obviously, we have

$$
-\left(a-b\left\|u_{*}\right\|^{2}\right) \Delta u_{*}=f(x) u_{*}^{p-1} .
$$

Combining with (2.10), we get

$$
-\Delta u_{*}=\frac{f(x) u_{*}^{p-1}}{a-b\left\|u_{*}\right\|^{2}} \geqslant 0
$$

Hence, by the strong maximum principle, one has $u_{*}>0$, that is, $u_{*}$ is a positive local minimal solution of problem (1.1). Thus, the proof of Proposition 2.2 is completed.

Proposition 2.3. Assume $1 \leqslant p<2,2<p<2^{*}$ or $p=2,|f|_{\frac{2^{*}}{2^{*}-2}}<a S$, and $f \in L^{\frac{2^{*}}{2^{*}-p}}(\Omega)$ is nonzero and nonnegative. Then (1.1) has at least one positive mountain-pass solution $\mathfrak{u}_{* *}$ with $\mathrm{I}\left(\mathfrak{u}_{* *}\right)>0$. 
Proof. We claim that I satisfies the mountain-pass geometry in $\mathrm{H}_{0}^{1}(\Omega)$. Firstly, we should prove that there exists $e \in \mathrm{H}_{0}^{1}(\Omega)$ with $\|e\|>\mathrm{R}$ such that $\mathrm{I}(e)<0$. In fact, fixing $\mathrm{u} \in \mathrm{H}_{0}^{1}(\Omega)$ and $u \neq 0$, as $\mathrm{t} \rightarrow+\infty$, one has

$$
I(t u)=\frac{a t^{2}}{2}\|u\|^{2}-\frac{b t^{4}}{4}\|u\|^{4}-\frac{t^{p}}{p} \int_{\Omega} f(x)|u|^{p} d x \rightarrow-\infty,
$$

which implies that there exists $e \in \mathrm{H}_{0}^{1}(\Omega)$ with $\|e\|>\mathrm{R}$ such that $\mathrm{I}(e)<0$. Secondly, we prove that there exist $R, \rho>0$ such that $\left.I(u)\right|_{u \in S_{R}} \geqslant \rho$. When $1 \leqslant p<2$, from Proposition 2.2, there exist $T, R, \rho>0$, for every $|\mathrm{f}|_{2^{2^{*}}-\mathrm{p}}<\mathrm{T}$ such that $\left.\mathrm{I}(\mathrm{u})\right|_{\mathfrak{u} \in S_{\mathrm{R}}} \geqslant \rho$. While $\mathrm{p}=2,|\mathrm{f}|_{\frac{2^{*}}{2^{*}-2}}<\mathrm{aS}$, we can easily obtain this conclusion by the similar way. When $2<p<2^{*}$, obviously, 0 is a local minimizer of I with $\mathrm{I}(0)=0$. In fact, fixing $\mathfrak{u}_{0} \in \mathrm{H}_{0}^{1}(\Omega)$ and $\mathfrak{u}_{0} \neq 0$, we have

$$
\lim _{t \rightarrow 0^{+}} \frac{I\left(t u_{0}\right)}{t^{2}}=\frac{a}{2}\left\|u_{0}\right\|^{2}>0,
$$

which implies that there exist $R, \rho>0$ such that $\left.I(u)\right|_{u \in S_{R}} \geqslant \rho$. Hence, our claim is proved to be true. Taking

$$
\mathrm{c}_{0}=\inf _{\gamma \in \Gamma} \max _{\mathrm{t} \in[0,1]} \mathrm{I}(\gamma(\mathrm{t})),
$$

where $\Gamma=\left\{\gamma \in \mathrm{C}\left([0,1], \mathrm{H}_{0}^{1}(\Omega)\right): \gamma(0)=0, \gamma(1)=e\right\}$. We claim that $\mathrm{c}_{0} \leqslant \frac{\mathrm{a}^{2}}{4 \mathrm{~b}}$. In fact,

$$
\begin{aligned}
\max _{t \in[0,1]} I(t e) & =\max _{t \in[0,1]}\left(\frac{a t^{2}}{2}\|e\|^{2}-\frac{b t^{4}}{4}\|e\|^{4}-\frac{t^{p}}{p} \int_{\Omega} f(x)|e|^{p} d x\right) \\
& \leqslant \max _{t \in[0,1]}\left(\frac{a t^{2}}{2}\|e\|^{2}-\frac{b t^{4}}{4}\|e\|^{4}\right) \\
& \leqslant \frac{a^{2}}{4 b} .
\end{aligned}
$$

Therefore, by Lemma 2.1, applying the mountain-pass lemma, there exists $u_{* *} \in H_{0}^{1}(\Omega)$ such that $I\left(u_{* *}\right)=$ $c_{0}>0$, that is, $u_{* *}$ is a nonzero mountain-pass solution of $(1.1)$. Since $I(u)=I(|u|)$, from [1, Theorem 10], one has $u_{* *} \geqslant 0$ in $\Omega$. Similar to Proposition 2.2 , by the strong maximum principle, we can prove that $\mathfrak{u}_{* *}$ is a positive mountain-pass solution of (1.1). This completes the proof of Proposition 2.3.

According to Proposition 2.2 and Proposition 2.3, we can obtain the proof of Theorem 1.1.

\section{Acknowledgment}

This work is supported by the Natural Science Foundation of Education of Guizhou Province (No. KY[2016]281, KY[2017]297); Science and Technology Foundation of Guizhou Province (No. LH[2015]7595, LH[2016]7054).

\section{References}

[1] H. Berestycki, I. Capuzzo-Dolcetta, L. Nirenberg, Variational methods for indefinite superlinear homogeneous elliptic problems, NoDEA Nonlinear Differential Equations Appl., 2 (1995), 553-572. 2

[2] C.-Y. Lei, C.-M. Chu, H.-M. Suo, Positive solutions for a nonlocal problem with singularity, Electron. J. Differential Equations, 2017 (2017), 9 pages. 1

[3] C.-Y. Lei, J.-F. Liao, H.-M. Suo, Multiple positive solutions for nonlocal problems involving a sign-changing potential, Electron. J. Differential Equations, 2017 (2017), 8 pages. 1, 1.2

[4] T.-F. Wu, On semilinear elliptic equation involving concave-convex nonlinearities and sign-changing weight function, J. Math. Anal. Appl., 318 (2006), 253-270. 2

[5] G.-S. Yin, J.-S. Liu, Existence and multiplicity of nontrivial solutions for a nonlocal problem, Bound. Value Probl., 2015 (2015), 7 pages. $1,1.2$ 